\title{
Earthquake Disaster Emergency Logistics of Transport Route Optimization Research
}

\author{
Dai Jinhui \\ Academic Affairs Office \\ Shengyang Aerospace University \\ Shengyang China \\ daijinhui@sau.edu.cn
}

\begin{abstract}
In recent years the earthquake happens frequently, China is one of the countries that suffer the most. The earthquake caused great harm to the national economy and the social development. Under this circumstance, it's necessary to have the research of route optimization in the earthquake.Through the analysis of the actual situation of earthquake disasters, this paper expounds the earthquake disaster emergency logistics of road transportation route choice effects, such as distribution lines, total delivery time, traffic volume, road traffic capacity and its safety factors influencing decision-making, and points out the goal that the earthquake disaster emergency logistics management needs to achieve. Then, the introduction of the minimum time and the maximum flow theory constructs the based model and determining the seismic disaster emergency logistics system highway transportation route choice the basic train of thought to clear the path and freight volume constraint conditions each other, established the earthquake disaster emergency logistics system highway transportation route choice model, and puts forward some solutions to this model algorithm. Finally, through an example, the paper validates the feasibility of the model and the algorithm.
\end{abstract}

Keyword-Earthquake disaster; Emergency logistics; Road transportation; Route optimization

\section{INTRODUCTION}

Since entering the new century, with the continuous development and progress of science and technology, human processing ability of emergencies has been greatly promoted. However, due to the unpredictability and endlessness of emergencies, we human still suffer extensive damages and great loss when it happens.

China has vast area and complicated topography, thus we are one of the most serious countries affected by the earthquake and other natural disasters in the world. In recent years, China's massive quake-prone areas which are widely distributed, which cause great damage to our country's social development, economic construction and people's life. When the earthquake occurs, divisions of government organs can quickly organize to set up the emergency disaster relief center, also with the human and material resources supported by other areas, our emergency response management system is still not perfect at present. Disasters cause serious damages to infrastructure such as road, railways and base station, which lead to the interruption of communication and transportation and the inside information of disaster area urgently needed by the emergency disaster relief command center unable to transfer out accurately and timely, and the relief supplies urgently needed in the disaster areas delivered accurately and timely. In order to reduce the loss caused by earthquake disaster, the relief supplies will have to be delivered as many as possible in the shortest possible time and in the most efficient way. It also requires the domestic experts and scholars to carry out the study of emergency logistics transportation route optimization concerning natural disaster, to put forward the scientific theory, to find the right way and to provide reference for China's earthquake relief work.

\section{OVERVIEW OF EMERGENCY LOGISTICS TRANSPORTATION ROUTE OPTIMIZATION THEORIES CONCERNING EARTHQUAKE DISASTER}

\section{A. Connotation of earthquake disaster emergency logistics}

Emergency logistics is the extension of logistics concept in the field of emergency disaster management, and is not a new form of logistics. In today's society, natural disasters and man-made emergencies occur frequently, so all the countries attach great importance to the research of emergency logistics.

On the basis of research on emergency logistics system, Wang Feng and others point out that the emergency logistics is a special logistics activity responds to demand for materials, personnel and funds from major natural disasters, terrorist attacks, public security, health emergencies, military conflicts and other emergencies.

And earthquake disaster emergency logistics is a general designation of all logistics activities around earthquake-relief work including relief materials collection, processing, packaging, storage and distribution. Earthquake disaster emergency logistics includes all the aspects from the disaster relief logistics equipment use and maintenance to the information collection, transmission and processing in the process of disaster relief. Earthquake disaster emergency logistics is different from ordinary logistics as time is the primary concern. The research content as shown in figure 1.

\section{B. Overview of transportation route optimization}

\section{1) Connotation of transportation route optimization}

Transportation is kind of physical activity realizing the variation of space position of people and objects. The logistics transportation refers to the process of carrying "objects" from one location to another location using vehicles, aircraft, ships and other transportation tools, which are changes of time and space state for "objects". 
Modern logistics believes transportation can create "place effect". Because the location is different, the same "object" brings different benefits; its own value-in-use for human beings is different, and this is so-called "place effect ". Transport the "object" from low "place effect" to high "place effect" can maximize the input-output ratio and ensure the rational allocation of resources. In this sense, I transportation improves its use value by changing the space position of "object". Therefore, optimizing the transportation route and reducing unnecessary consumption in transport process have important implications.

In general, transportation route optimization may be defined as: in order to optimize the whole logistics system, applying relevant principle and methods of systems engineering, establishing the mathematical model for logistics system based on operational research, selecting the appropriate means of transport, planning the reasonable transportation line, giving full play to the advantage of different transport ways, well-ordering the product transportation activities, making the transport with fewest links, lowest costs, shortest paths and fastest speed, avoiding the occurrence of unreasonable transportation situation.

2) Principle of transportation route selection

a) Principle of security

Security is the first consideration of transportation route selection; other targets of transportation route optimization are meaningful only in the case of ensuring the safety.

\section{b) The economy principle}

The goal and results of route optimization are diverse. No matter what goals to achieve, trying to reduce the transportation cost must be considered.

\section{c) The realistic principle}

Route of transport must be operable and practical; ignoring the difficulty of the actual operation and simply theory verification should be avoided. Follow the scientific basis; investigate and study the society deeply; a detailed investigation of transport routes and surrounding environment, selecting scientific and practical method according to the reality, method of choosing transport route should avoid being too trivial and time-taking, should be easy to understand, to learn, and to promote.

\section{d) The strategic principle}

In the process of transportation route selection, we should have strategic vision. Both the actual needs of the current situation and possibilities for future development should be fully considered.

\section{Overview of material transportation route optimization concerning earthquake disaster}

Material transportation route optimization concerning natural disaster first proposed by Fiedrich, is one of the branch of vehicle optimized scheduling problem, trying to quickly establish a certain amount of rescue sites in a short period of time after the disaster, planning corresponding rescue activities, and building dynamic vehicle transportation route optimization model.

Based on the above theories, the earthquake disaster material transportation route optimization in this paper is defined as: After the earthquake disasters, in the circumstances of infrastructure severely damaged, via reasonable arrangement of vehicle transport routes, the relief materials can be delivered in the shortest time and with the greatest amount to the affected.

Earthquake disaster emergency material distribution process is shown in the following figure 3 .

\section{MODEL OF EMERGENCY LOGISTICS TRANSPORTATION ROUTE OPTIMIZATION CONCERNING EARTHQUAKE DISASTER}

\section{A. Theoretical basis of model construction}

Transportation of relief supplies has its own particularity. In the process of relief materials transportation, the time, volume and other factors need to be considered ensuring that relief materials can be delivered in the shortest time and with the greatest amount to the affected, while the cost problems considered in the general transport can be neglected. That is a major distinction between the relief transport and general transport. In this paper, the study of the transportation route optimization of relief supplies introduces the "the minimum cost and maximum flow" problem in operational research, constructs "the shortest time and maximum flow" model, in the hope of giving a hand to earthquake disaster emergency management.

The problem of "the minimum cost and maximum flow" means that given a network $\mathrm{G}=(\mathrm{V}, \mathrm{E}, \mathrm{C})$, for each arc (vi, vj) $\in \mathrm{E}$, besides rated capacity cij, also the cost of the unit flow $\mathrm{d}(\mathrm{vi}, \mathrm{vj}) \geqslant 0$ (denoted as dij), seeking a maximum flow $\mathrm{f}$, minimizing the total cost of flow.

$$
d(f)=\sum_{\left(v_{i}, v_{j}\right) \in E} d_{i j} f_{i j}
$$

In fact, in a network $\mathrm{G}$ when an augmenting path $\mu$ along the feasible flow $\mathrm{f}$, adjusts the quantity $\theta=1$ to improve $f$, getting a new flow of feasible flow $f$ ', with $w\left(f^{\prime}\right)=w(f)+1$, while the total cost $b\left(f^{\prime}\right)$ is increased comparing with $b(f)$.

$$
\begin{aligned}
\sum_{\boldsymbol{\mu}^{+}} \boldsymbol{b}_{\boldsymbol{i} \boldsymbol{j}}-\sum_{\boldsymbol{\mu}-} \boldsymbol{b}_{\boldsymbol{i} \boldsymbol{j}}, \text { the cost of the augmenting } \\
\begin{aligned}
& \text { path. } \\
&\left(f^{\prime}\right)-b(f)=\sum_{\mu^{+}} b_{i j}\left(f_{i j}^{\prime}-f_{i j}\right)-\sum_{\mu^{-}} b_{i j}\left(f_{i j}-f_{i j}^{\prime}\right) \\
&=\sum_{\mu^{+}} b_{i j}-\sum_{\mu-} b_{i j}
\end{aligned}
\end{aligned}
$$

If the cost of feasible flow $\mathrm{f}$ is lowest in all feasible flow with the flow of $w(f)$ and $m$ has the lowest cost in all augmenting path on $f$. Adjust the feasible flow $f$ along augmenting path $\mu$ will get new feasible flow $\mathrm{f}^{\prime}$, which is the least flow cost in all the feasible flow with a flow of $\mathrm{w}\left(\mathrm{f}^{\prime}\right)$.

So on, when $\mathrm{f}^{\prime}$ is the maximum flow, which is also the required maximum flow with minimum cost.

The basic train of thought for solving minimum cost maximum flow:

Note that, due to $\mathrm{dij} \geqslant 0, \mathrm{f}=0$ must be the minimum cost flow when the flow is 0 . This can always start from $\mathrm{f}=$ 
0.Generally, set $\mathrm{f}$ always the minimum cost flow of flow $\mathrm{v}$ (f), the remaining problem is how to seek augmenting path of $\mathrm{f}$ with lowest cost. To do this, we can construct a weighted directed graph L (f) with the same vertex as the original network $G$, and change each arc (vi, vj) of $G$ into two $\operatorname{arcs}(v i, v j)$ and (vj, vi) with opposite directions. Define the weight lij in the arc of L (f) as:

$$
l_{i j}=\left\{\begin{array}{c}
d_{i j}, \text { when } f_{i j}<c_{i j} \\
+\infty, \text { whenf }_{i j}=c_{i j}
\end{array}\right.
$$

side $(v i$, vj $) \in E$

$\operatorname{side}(v j, v i)$ is the flip edge of $(v i, v j)$ in the original plot $\mathrm{G}$

$$
l_{j i}= \begin{cases}-d_{i j}, \text { when } f_{i j}>0 \\ +\infty, \text { whenf }_{i j}=0\end{cases}
$$

And remove the side with the weight $+\infty$.

So seeking the augmenting path the minimum cost in $\mathrm{G}$ net is equal to seek the shortest circuit from vs to vt in L(f ) length network. The basic steps:

(1) Value zero flow $\mathrm{f}(0)=\{0\}$

(2) If get the minimum cost flow $\mathrm{f}(\mathrm{K}-1)$ in the step of $\mathrm{K}-1$, construct a weighted directed graph L(f (k-1)), and seek the shortest circuit from vs to vt .

(3) If there is no the shortest circuit (the power of the shortest circuit is infinite), then $\mathrm{f}(\mathrm{k}-1)$ is minimum cost maximum flow. If there is, get the corresponding augmenting path in the original network $\mathrm{G}$.

(4) Adjust the(f (k-1)) on the augmenting path $\mu$, value the adjusted.

$$
\begin{gathered}
\theta=\min \left\{\min _{\mu^{+}}\left(c_{i j}-f_{i j}^{(k-1)}\right), \min _{\mu^{-}}\left(f_{i j}^{(k-1)}\right)\right\} \\
\text { Make } f_{i j}^{(k)}=\left\{\begin{array}{cc}
f_{i j}^{(k-1)}+\theta, & \text { at } \mu^{+} \\
f_{i j}^{(k-1)}-\theta, & \text { at } \mu^{-} \\
\text {othersfixed }
\end{array}\right.
\end{gathered}
$$

\section{B. The basic thoughts of model construction}

Highway transportation with strong flexibility is the best way to transport relief supplies, and adapt to different environment. From collecting relief supplies to distributing them to victims, the road transport almost entirely undertakes the supplies shift.

Highway system's ability is given full play, which can satisfy the demands in the disaster areas to the utmost extent and be very beneficial for rescue work. The key to the highway transportation route optimization is how to deliver the most relief supplies within the shortest time to the quake-hit regions. But in reality, when the earthquake hit, it will bring damaged bridges, road congestion and other infrastructure destroyed, then the transportation network suffers severe damage, and the normal road can't go smoothly. The phenomenon such as vehicle detour, return, unable to reach the hit area appears. Rescue workers can't get to the disaster area timely for the earthquake relief work, and relief supplies cannot be delivered into the disaster area, which seriously affect the rescue work. At this time the problem of how to ensure the shortest highway transport with biggest volume of relief supplies becomes very important. In view of the huge role played by the road transportation in the process of transporting relief supplies after the disaster, the paper chooses highway transportation as the research object.

\section{Model construction of emergency logistics transportation route optimization concerning earthquake disaster}

\section{1) Relevant assumption}

The model of "the shortest time and maximum flow" constructed in this paper is to find the inner link among the maximum capacity, time and traffic volume, and then to quantify and identify the time and traffic volume. In order to clearly illustrate the relationship between these variables, we make the following assumptions:

The vehicles move at a constant speed on all roads; due to different distances of the road in each section, therefore, the time is different.

Adopting the vehicles with the same specifications and carrying capacity, the inflow and outflow of each node are integer times of the vehicle load.

The maximum road capacity is limited, and the traffic volume should not be out of limits.

The entry quantity of vehicles is equal to the outflow volume on each node, and its total inflow is equal to the total outflow for the entire transportation network.

2) Model construction

According to the above model assumption, we got the effect factors involved, therefore, the basic variable of model sets:

VI - on behalf of the nodes in the network

$\mathrm{Cij}$ - signify the maximum capacity of

Dij - signify the travel time of

for the above assumptions, the problem can be transformed into a network model. The directed graph $G$ $(\mathrm{V}, \mathrm{E})$, in which $\mathrm{V}$ is for vertex set, that is the starting point, end point in the road and middle road intersection; , on behalf of the arc set; arc says there's a path between two vertexes; , signifies the capacity of arc , that is, the maximum vehicle capacity of the road during a period of time; , signifies the time of passing arc. The question becomes network optimization model, by solving the start point and end point, ( signifies the flow passing the arc ), and makes the time for $\mathrm{f}$ passing the network shortest.

To sum up, the main purpose of the model construction is to choose the appropriate transportation paths for relief supplies, to maximize the traffic volume and minimize the spending time under the condition of meeting the allowed maximum traffic volume on all feasible roads. Based on the above conditions, the objective function is:

\section{3) Model algorithm}

According to the theory of minimum cost maximum flow, the above model algorithm can be summarized as: in the earthquake disaster emergency network $\mathrm{G}=(\mathrm{V}, \mathrm{E}, \mathrm{C}$, D) ( $\mathrm{V}$ for vertex set, i.e. starting point and end point in the road and middle road intersection); is on behalf of the 
arc set, i.e. the road between each vertex; where there is an arc, there is passable road between two vertex; , says the capacity of the arc, i.e. the maximum traffic volume of the roads; , says the time passing the arc. If the cost of feasible flow $f$ is lowest in all feasible flow with the flow of l(f ) and $m$ has the lowest cost in all augmenting path on f. Adjust the feasible flow f along augmenting path $\mu$ will get new feasible flow $\mathrm{f}^{\prime}$, which is the least flow cost in all the feasible flow with a flow of $1\left(f^{\prime}\right)$.

So on, when $f^{\prime}$ is the maximum flow, which is also the required maximum flow with minimum duration.

The algorithm process of minimum duration maximum flow is shown in figure 4.

Value zero flow $\mathrm{f}(0)=\{0\}$

If get the minimum cost flow $\mathrm{f}(\mathrm{K}-1)$ in the step of $\mathrm{K}-1$, construct a weighted directed graph $\mathrm{L}(\mathrm{f}(\mathrm{k}-1))$, and seek the shortest circuit from vs to vt .

If there is no the shortest circuit (the power of the shortest circuit is infinite), then $\mathrm{f}(\mathrm{k}-1)$ is minimum cost maximum flow. If there is, get the corresponding augmenting path in the original network $\mathrm{G}$.

Adjust the(f $(k-1))$ on the augmenting path $\mu$, value the adjusted.

$$
\begin{aligned}
& \theta=\min \left\{\min _{\mu^{+}}\left(c_{i j}-f_{i j}^{(k-1)}\right), \min _{\mu^{-}}\left(f_{i j}^{(k-1)}\right)\right\} \\
& \theta=\min \left\{\min _{\mu^{+}}\left(c_{i j}-f_{i j}^{(k-1)}\right), \min _{\mu^{-}}\left(f_{i j}^{(k-1)}\right)\right\}
\end{aligned}
$$

Make

$$
f_{i j}^{(k)}=\left\{\begin{aligned}
f_{i j}^{(k-1)}+\theta, & \text { at } \mu^{+} \\
f_{i j}^{(k-1)}-\theta, & \text { at } \mu^{-} \\
\text {othersfixed } &
\end{aligned}\right.
$$

\section{CONCLUSION}

In modern times, all kinds of natural disasters, terrorist attacks, military conflicts and other man-made accidents occur frequently. The research of emergency logistics will become a major focus in the academic circles. Emergency logistics management involves many aspects of society; our country has not set up perfect emergency logistics management system, so the research on emergency logistics is very necessary.

This paper first describes the background of emergency logistics emerging, and then states the purpose and significance of the study. Secondly, it introduces related theories of the earthquake disaster emergency logistics and transport route optimization, then analyzes the characteristics of earthquake disaster emergency logistics and contents and existing problems of emergency logistics management, and clarifies that the research of earthquake disaster emergency logistics is of great importance. Through the approach of combining qualitative analysis with quantitative analysis, data modeling with example analysis, consulting a large number of relevant materials, applying relevant theories and methods of operational research, the paper studies the highway transportation route optimization of relief supplies concerning the natural disasters in the earthquake. The paper introduces the shortest time maximum flow theory, clarifies the basic idea of material transportation route optimization in earthquake disaster emergency logistics management, determines the relationship between transport time and total transport volume, constructs the basic model of transportation routeoptimization in earthquake disaster emergency logistics systems, and puts forward the algorithm of the model.

\section{REFERENCES}

[1] Zhao Tong, Fan Houming, Wang Guilin, etc. Route optimization model for emergency aid supplies distribution vehicles

[2] Logistics technology, 2010,10(227),63-68

[3] Chou Ge. The study of route problems concerning emergency logistics management [D].Chengdu: Southwest Jiaotong University, 2010.

[4] Ke Ke. The research on the emergency logistics distribution route optimization [J]. Logistics engineering and management: 2011, 7(7): 63-65

[5] Zheng Liqun, Ji Kaiqing, Li Dawei. The model research on materia distribution in emergency supplies demand area [D]. Liaoning: Liaoning University of science and technology, 2009.

[6] Bai Yongxiu, Zhou Xizhao. The research on emergency supplies distribution route optimization problems [J]. Logistics technology: 2009, 28(3):99-91.

[7] Zhou Zuoshan. Research on the framework of disaster logistics system and material distribution model [D]. Wuhan: Huazhong University of Science and Technology, 2009

[8]Paessens $H$. The Saving Algorithm for the Vehicle Problem[J] . European Journal of Operational Research , 1988 , $34: 336-344$.

[9] Baker B , A . Yechew . A Genetic Algorithm for the Vehicle Routing Problem[J] . Computers OperationsResearch2003 , 30(2) : 787-800 


\begin{tabular}{|c|r|c|}
\hline \multirow{2}{*}{ transport connections, route and means of transport in logistics network } \\
\hline \multirow{2}{*}{ Station } & Bus, airplane & Station \\
Logistics center & Ship, railway, pipeline & Logistics center \\
\cline { 2 - 2 } Port & Means & Port \\
Airport & Airport \\
Yard & Highway, waterway, pipe \\
network, airline, railway & Yard \\
\cline { 2 - 2 } Transport connections & Transport connections \\
\cline { 2 - 2 } & Transport & \\
\hline
\end{tabular}

Figure 1. Diagram of transport network

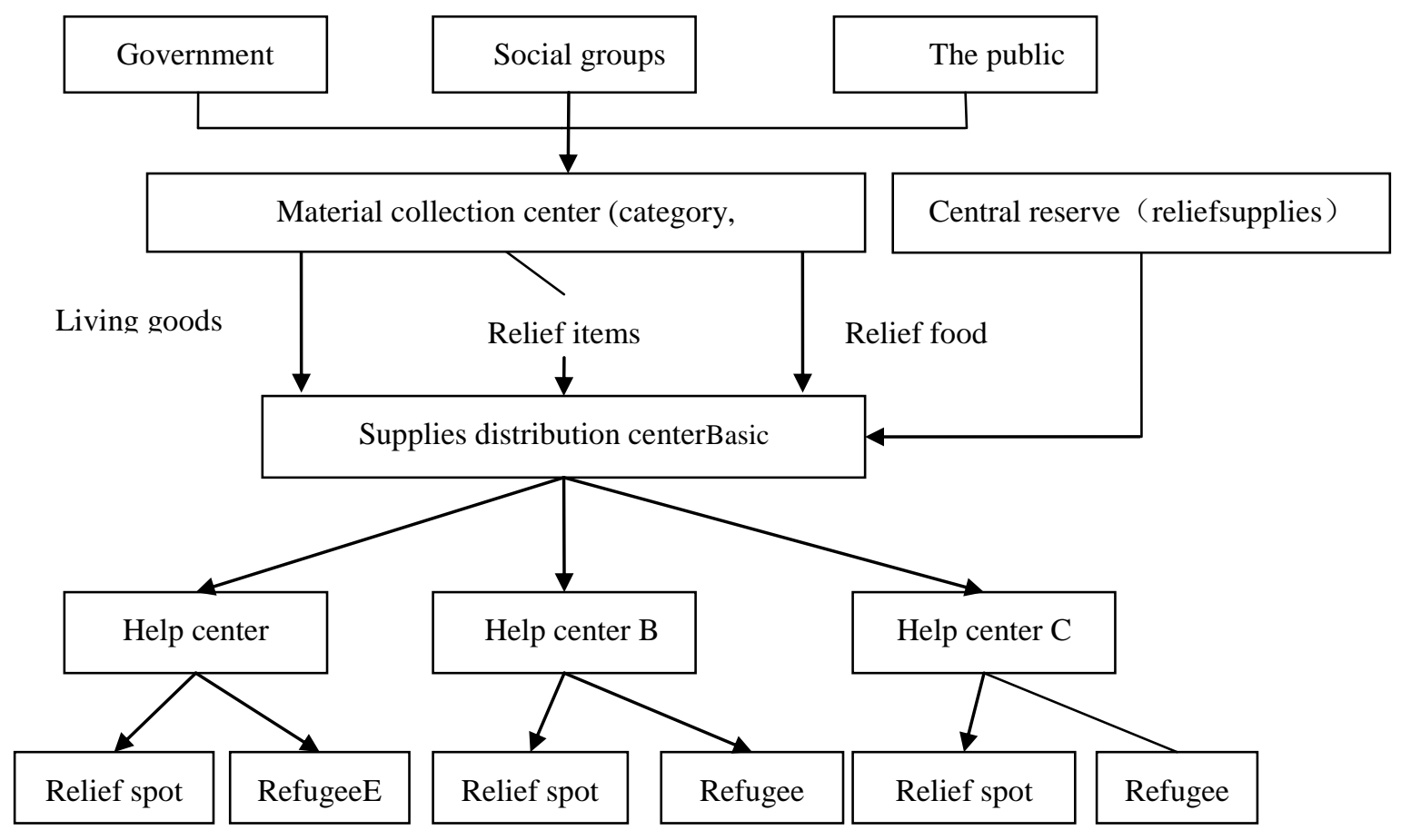

Figure 2. Earthquake disaster emergency material distribution process 


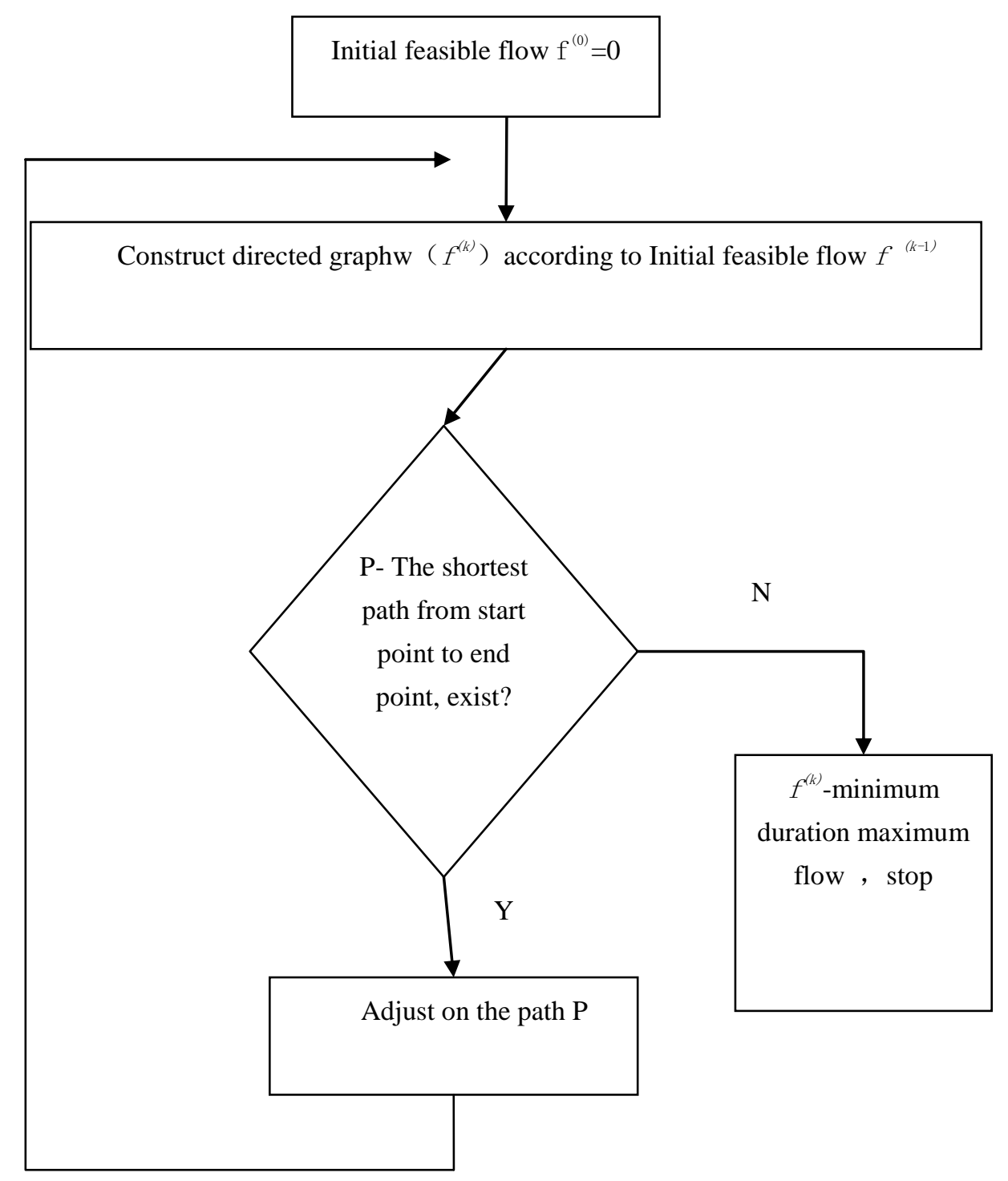

Figure 3. Flow chart of minimum duration maximum flow 